DOI 10.4171/JEMS/242

Guy Barles · Emmanuel Chasseigne · Cyril Imbert

\title{
Hölder continuity of solutions of second-order non-linear elliptic integro-differential equations
}

Received January 12, 2008 and in revised form May 21, 2008

\begin{abstract}
This paper is concerned with Hölder regularity of viscosity solutions of second-order, fully non-linear elliptic integro-differential equations. Our results rely on two key ingredients: first we assume that, at each point of the domain, either the equation is strictly elliptic in the classical fully non-linear sense, or (and this is the most original part of our work) the equation is strictly elliptic in a non-local non-linear sense we make precise. Next we impose some regularity and growth conditions on the equation. These results concern a large class of integro-differential operators whose singular measures depend on $x$ and also a large class of equations, including Bellman-Isaacs equations.
\end{abstract}

Keywords. Hölder regularity, integro-differential equations, Lévy operators, general non-local operators, viscosity solutions

\section{Introduction}

The aim of this paper is to show that viscosity solutions of fully non-linear elliptic integrodifferential equations are Hölder continuous under general suitable strict ellipticity and regularity/growth conditions on the equations. We also obtain explicit $C^{0, \alpha}$ estimates in terms of the space dimension, the non-linearity, the singular measure and the (local) $L^{\infty}$. bound of the solution.

To be more specific, we describe the general framework we consider. We are interested in equations of the type

$$
F\left(x, u, D u, D^{2} u, \mathcal{I}[x, u]\right)=0 \quad \text { in } \Omega
$$

where $\Omega$ is a domain in $\mathbb{R}^{N}$ (not necessarily bounded) and $\mathcal{I}[x, u]$ is an integro-differential operator. The function $u$ is real-valued and $D u, D^{2} u$ stand respectively for its gradient and Hessian matrix. The non-linearity $F$ is a (continuous) function which is degenerate elliptic: in this context, this means that $F$ is non-increasing with respect to

G. Barles, E. Chasseigne: Laboratoire de Mathématiques et Physique Théorique CNRS UMR 6083, Fédération Denis Poisson, Université François Rabelais, Parc de Grandmont, 37200 Tours, France; e-mail: barles@lmpt.univ-tours.fr, emmanuel.chasseigne@lmpt.univ-tours.fr

C. Imbert: CEREMADE, UMR CNRS 7534, Université Paris-Dauphine, Place de Lattre de Tassigny, 75775 Paris Cedex 16, France; e-mail: imbert@ceremade.dauphine.fr

Mathematics Subject Classification (2010): Primary 35D99, 35J60, 35B05, 47G20 
its last two variables (see below for a precise statement and [4] for further details). The integro-differential operators we will consider in the present paper are defined as follows:

$$
\mathcal{I}[x, u]=\int_{\mathbb{R}^{N}}\left(u(x+z)-u(x)-D u(x) \cdot z \mathbf{1}_{B}(z)\right) \mu_{x}(d z)
$$

where $\mathbf{1}_{B}$ denotes the indicator function of the unit ball $B$ and $\left\{\mu_{x}\right\}_{x \in \mathbb{R}^{N}}$ is a family of Lévy measures, i.e. non-negative, possibly singular, Borel measures on $\mathbb{R}^{N}$ such that

$$
\int_{\mathbb{R}^{N}} \min \left(|z|^{2}, 1\right) \mu_{x}(d z)<+\infty .
$$

We point out that the solution $u$ has to be given in the whole space $\mathbb{R}^{N}$ even if $(1.1)$ is satisfied on a domain $\Omega$; see Section 2 for further details.

Important "special cases" of operators of the form (1.2) are Lévy-Itô operators, namely

$$
\mathcal{I}_{L I}[x, u]=\int_{\mathbb{R}^{N}}\left(u(x+j(x, z))-u(x)-D u(x) \cdot j(x, z) \mathbf{1}_{B}(z)\right) \mu(d z)
$$

where $\mu$ is a Lévy measure and $j(x, z)$ is the size of the jumps at $x$. For the operator to be well-defined, one usually assumes

$$
|j(x, z)| \leq C_{0}|z| \quad \text { for some constant } C_{0} \text { and for any } x \in \Omega, z \in \mathbb{R}^{N} .
$$

Model examples for a Lévy measure $\mu$ and jump function $j$ are the ones associated with the fractional Laplacian: $\mu(d z)=d z /|z|^{N+\alpha}$ (with $0<\alpha<2$ ) and $j(x, z)=z$. This class of operators appears in the context of stochastic control of jump processes and it is also worth pointing out that, at least to the best of our knowledge, general comparison results (for viscosity solutions) are only available for operators in the form 1.4.

Many papers deal with Hölder estimates for fully non-linear elliptic equations. There are two kinds of approaches: for uniformly elliptic equations, one can use the powerful approach by Harnack inequalities which leads also to further regularity results; we refer the reader to Cabré and Caffarelli [7] or Trudinger [16, 17] and references therein for results in this direction. A simpler method, more closely related to classical viscosity solutions theory, was introduced by Ishii and Lions in [13]. Besides its simplicity, it has the further advantage of providing results under weaker ellipticity assumptions and even for some degenerate equations; it was used in [1], [10] where further regularity results are also proved and later in [5, 3]. As far as integro-differential elliptic equations are concerned, many papers were published in potential theory, with equations being linear in most of those papers; moreover they rely on probabilistic techniques. See for instance [6]. One of the first papers about Hölder estimates for integro-differential equations with PDE techniques is probably [15]. It mainly deals with linear equations where singular measures $\mu_{x}$ have a very general $x$-dependence, improving on the previous literature; it also deals with quite particular non-linear equations, more precisely, $F(\mathcal{I}[x, u], \mathcal{J}[x, u])=0$ where $\mathcal{I}$ and $\mathcal{J}$ are two different non-local terms, and for "strictly elliptic" functions $F$ (in a suitable sense).

In the present paper, we deal with fully non-linear elliptic equations and we obtain local $C^{0, \alpha}$ regularity and estimates for a quite general class of integro-differential operators 
of the form (1.2) satisfying proper assumptions (see (3.2)-(3.4)). Even if important operators (1.4) can be seen as special cases of the general one, we will give specific results with specific assumptions. In other words, our second main theorem is not a corollary of the first one.

Let us mention that, on the one hand, we do not cover all the examples given in [15] (in particular those in Section 3.4 of [15]), but on the other hand, we can treat examples outside the scope of [15]; indeed, we only assume that the measure $\mu_{x}$ is bounded at infinity (uniformly with respect to $x$ ), while condition (2.2) of [15] requires a (small) power of $|z|$ to be integrable at infinity. Moreover, we can handle much more general non-linear equations, including the important Bellman-Isaacs equations, and we can also identify the critical Hölder exponent $\alpha$ of the solution. To be more precise, we are able to prove that the solution is $\alpha$-Hölder continuous for any $\alpha<\beta$ (and even $\alpha=\beta$ under stronger assumptions in the case $\beta<1$ ) where $\beta$ characterizes the singularity of the measure associated with the integral operator.

In order to treat a large class of non-linear elliptic equations, we decided to present the main results by assuming that the non-linearity $F$ in 1.1 satisfies a proper ellipticitygrowth condition (see $(\mathrm{H})$ in Section 3 . Loosely speaking, this structure condition ensures that either the equation is strictly elliptic in the classical fully non-linear sense or it is strictly elliptic in a non-linear and non-local sense. Since this condition is rather technical, a whole section is devoted to comments and examples (see Section 47).

The techniques we use in the present paper do not seem to yield Lipschitz regularity when $\beta \geq 1$ and we intend to investigate this question in a future work. Let us mention that we proved [2] in quite a general framework that there exists a viscosity solution of the Dirichlet problem without loss of the boundary condition. The local Hölder estimate we obtain in the present paper applies to the Dirichlet problem too and we will address naturally in a future work the question of boundary estimates. We also point out that the techniques we develop here can be readily applied to parabolic integro-differential equations. Finally, another possible interesting application of these results is the study of the ergodicity of non-local equations.

We conclude this introduction by mentioning that after this work was completed, we found out that Caffarelli and Silvestre [8] obtained regularity results for a large class of non-linear integro-differential equations that are invariant under $x$-translations and uniformly elliptic in a non-local way. In particular, they are able to get $C^{1, \alpha}$ regularity of solutions.

The paper is organized as follows. In Section 2 we recall the definition of a viscosity solution of (1.1). In Section 3, we state two main results: the first one deals with a general $x$-dependent Lévy measure and the second with integro-differential operators in the Lévy-Itô form. In Section 4, we comment on the main structure assumption on the non-linearity $F$ and we give examples of direct applications of our results. Section 5 is devoted to the proofs of the main results.

Notation. The scalar product in $\mathbb{R}^{N}$ is denoted by $x \cdot y$. A ball centered at $x$ of radius $r$ is denoted by $B(x, r)$. If $x=0$, we simply write $B_{r}$, and if $r=1$, we even write $B$. Moreover, $\mathcal{S}^{N-1}$ denotes the unit sphere of $\mathbb{R}^{N}$. 
The transpose of a matrix $A$ is denoted $A^{*}$ and $\|A\|$ stands for the usual norm of $A$, namely $\|A\|:=\max _{|z|=1}|A z| . \mathbb{S}^{N}$ is the space of $N \times N$ real symmetric matrices. We recall that $X \geq Y$ if all the eigenvalues of $X-Y$ are non-negative. If $X \in \mathbb{S}^{N}$ and $\varepsilon \in(0,1)$ is such that all the eigenvalues of $X$ are strictly less than 1 (resp. strictly greater than -1$)$, we set $X^{\varepsilon}=(I-\varepsilon X)^{-1} X$ (resp. $\left.X_{\varepsilon}=(I+\varepsilon X)^{-1} X\right)$. These matrices are obtained from $X$ by applying a sup-convolution procedure (resp. an inf-convolution procedure), namely, for any $\xi \in \mathbb{R}^{N}$,

$$
X^{\varepsilon} \xi \cdot \xi=\sup _{\zeta \in \mathbb{R}^{N}}\left\{X \zeta \cdot \zeta-\frac{|\xi-\zeta|^{2}}{\varepsilon}\right\}, \quad X_{\varepsilon} \xi \cdot \xi=\inf _{\zeta \in \mathbb{R}^{N}}\left\{X \zeta \cdot \zeta+\frac{|\xi-\zeta|^{2}}{\varepsilon}\right\}
$$

\section{Viscosity solutions for PIDE}

In this section, we recall the notion of degenerate ellipticity for non-linear non-local equations and the definition of viscosity solutions for such equations.

\subsection{Degenerate ellipticity}

Throughout the paper, the domain $\Omega$ is an open subset of $\mathbb{R}^{N}$ and the non-linearity $F$ is a continuous function. We also assume that (1.1) is degenerate elliptic. In this framework, this means that we make the following

Assumption (E). For any $x \in \mathbb{R}^{N}, u \in \mathbb{R}, p \in \mathbb{R}^{N}, X, Y \in \mathbb{S}^{N}, l_{1}, l_{2} \in \mathbb{R}$,

$$
F\left(x, u, p, X, l_{1}\right) \leq F\left(x, u, p, Y, l_{2}\right) \quad \text { if } X \geq Y, l_{1} \geq l_{2} .
$$

\subsection{Non-local operators}

In order to define viscosity solutions for 1.1 , we introduce two associated operators $\mathcal{I}^{1, \delta}$ and $\mathcal{I}^{2, \delta}$ by

$$
\begin{aligned}
& \mathcal{I}^{1, \delta}[x, p, u]=\int_{|z|<\delta}\left[u(x+z)-u(x)-p \cdot z \mathbf{1}_{B}(z)\right] \mu_{x}(d z), \\
& \mathcal{I}^{2, \delta}[x, p, u]=\int_{|z| \geq \delta}\left[u(x+z)-u(x)-p \cdot z \mathbf{1}_{B}(z)\right] \mu_{x}(d z) .
\end{aligned}
$$

In the case of Lévy-Itô operators $1.4, \mathcal{I}^{1, \delta}$ and $\mathcal{I}^{2, \delta}$ are defined as follows:

$$
\begin{aligned}
& \mathcal{I}^{1, \delta}[x, p, u]=\int_{|z|<\delta}\left[u(x+j(x, z))-u(x)-p \cdot j(x, z) \mathbf{1}_{B}(z)\right] \mu(d z), \\
& \mathcal{I}^{2, \delta}[x, p, u]=\int_{|z| \geq \delta}\left[u(x+j(x, z))-u(x)-p \cdot j(x, z) \mathbf{1}_{B}(z)\right] \mu(d z) .
\end{aligned}
$$




\subsection{Definition}

We now recall the definition of a viscosity solution for 1.1. We assume that we are given a function $u$ defined on the whole space $\mathbb{R}^{N}$.

Definition 2.1 (Viscosity solutions). An upper semi-continuous (usc for short) function $u: \mathbb{R}^{N} \rightarrow \mathbb{R}$ is a subsolution of $\left[1.1\right.$ if for any test function $\phi \in C^{2}(B(x, \delta))$ such that $u-\phi$ attains a maximum on $B(x, \delta)$ at $x \in \Omega$,

$$
F\left(x, u(x), D \phi(x), D^{2} \phi(x), \mathcal{I}^{1, \delta}[x, p, \phi]+\mathcal{I}^{2, \delta}[x, p, u]\right) \geq 0
$$

where $p=D \phi(x)$.

A lower semi-continuous (lsc for short) function $u: \mathbb{R}^{N} \rightarrow \mathbb{R}$ is a supersolution of 1.1 if for any test function $\phi \in C^{2}\left(\mathbb{R}^{N}\right)$ such that $u-\phi$ attains a maximum on $B(x, r)$ at $x \in \Omega$ for some $r>0$,

$$
F\left(x, u(x), D \phi(x), D^{2} \phi(x), \mathcal{I}^{1, r}[x, p, \phi]+\mathcal{I}^{2, r}[x, u]\right) \leq 0,
$$

where $p=D \phi(x)$.

A continuous function $u: \mathbb{R}^{N} \rightarrow \mathbb{R}$ is a solution of (1.1) if it is both a subsolution and a supersolution.

Remark 2.2. It is possible to construct solutions of 11.1 in the case where $\Omega=\mathbb{R}^{N}$. If $\Omega \neq \mathbb{R}^{N}$, boundary conditions must be imposed. For instance, as far as the Dirichlet problem is concerned, the function $u$ can be prescribed outside of $\Omega$. See [2] for further details.

Remark 2.3. As remarked in [4], one can choose $r=0$ in the previous definition, at least in the case of Lévy-Itô operators. See [4] for further details.

\section{Main results}

In this section, we state the two main results of this paper: the first one is concerned with non-local operators of the form (1.2) (Theorem 3.1) and the second one provides other results for Lévy-Itô operators (1.4) (Theorem 3.2).

The two results rely on a structure condition imposed on the non-linearity $F$. In order to formulate it, we consider two functions $\Lambda_{1}, \Lambda_{2}: \bar{\Omega} \rightarrow[0,+\infty)$ such that $\Lambda_{1}(x)+$ $\Lambda_{2}(x) \geq \Lambda_{0}>0$ on $\bar{\Omega}$.

(H) (Ellipticity-growth condition) For any $R>0$, there exist constants $k \geq 0, \tau, \theta \in$ $(0,1]$, a locally bounded function $\chi: \mathbb{R}^{+} \times \mathbb{R}^{+} \rightarrow \mathbb{R}^{+}$, a modulus of continuity $\omega_{F}:(0,+\infty) \rightarrow(0,+\infty)$ with $\omega_{F}(0+)=0$ and two constants $\eta, \bar{\varepsilon}_{0}>0$ such that 
for any $x, y \in \Omega$ with $|x-y| \leq \eta, u, v \in \mathbb{R}$ with $|u|,|v| \leq R, p, q \in \mathbb{R}^{N},|q| \leq R$, $l_{1}, l_{2} \in \mathbb{R}$ with $l_{1} \leq l_{2}, \varpi \in(0,1 / 3), L>0, \bar{\varepsilon} \in\left(0, \bar{\varepsilon}_{0}\right)$ and $\hat{a} \in \mathcal{S}^{N-1}$, we have

$$
\begin{aligned}
& F\left(y, u, p, Y, l_{2}\right)-F\left(x, v, p+q, X, l_{1}\right) \\
& \leq \Lambda_{1}(x)\left(\operatorname{Tr}(X-Y)+\frac{\omega_{F}(|x-y|)}{\bar{\varepsilon}}+|x-y|^{\tau}|p|^{2+\tau}+|p|^{2}+\chi(L, \eta)\right) \\
& \quad+\Lambda_{2}(x)\left(\left(l_{1}-l_{2}\right)+\frac{|x-y|^{2 \theta}}{\bar{\varepsilon}}+|x-y|^{\tau}|p|^{k+\tau}+C_{F}|p|^{k}+\chi(L, \eta)\right)
\end{aligned}
$$

if the matrices $X, Y$ satisfy

$$
-\frac{4}{\bar{\varepsilon}} I \leq\left[\begin{array}{rr}
X & 0 \\
0 & -Y
\end{array}\right] \leq \frac{2}{\bar{\varepsilon}}\left[\begin{array}{rr}
Z & -Z \\
-Z & Z
\end{array}\right]+L\left[\begin{array}{ll}
I & 0 \\
0 & 0
\end{array}\right],
$$

where $I$ denotes the identity matrix and $Z=I-(1+\varpi) \hat{a} \otimes \hat{a}$.

In the next subsection, we comment on this structure condition and give several examples. The general results are the following.

Theorem 3.1 (Hölder continuity for general non-local operators). Assume that the measures $\mu_{x}$ satisfy the following: there exist $\beta \in(0,2)$, a constant $\tilde{C}_{\mu}>0$, a modulus of continuity $\omega_{\mu}:(0,+\infty) \rightarrow(0,+\infty)$ with $\omega_{\mu}(0+)=0$ and, for $\eta \in(0,1)$, a constant $C_{\mu}(\eta)>0$ such that for any $x, y \in \Omega, d \in \mathcal{S}^{N-1}$ and $\eta, \delta \in(0,1)$,

$$
\begin{aligned}
& \int_{B}|z|^{2} \mu_{x}(d z)+\int_{\mathbb{R}^{N} \backslash B} \mu_{x}(d z) \leq \tilde{C}_{\mu}, \\
& \int_{\{z:|z| \leq \delta,|d \cdot z| \geq(1-\eta)|z|\}}|z|^{2} \mu_{x}(d z) \geq C_{\mu}(\eta) \delta^{2-\beta}, \\
& \int_{B_{\delta}}|z|^{2}\left|\mu_{x}-\mu_{y}\right|(d z) \leq \omega_{\mu}(|x-y|) \delta^{2-\beta}, \\
& \int_{B \backslash B_{\delta}}|z|\left|\mu_{x}-\mu_{y}\right|(d z) \leq \begin{cases}\omega_{\mu}(|x-y|) \delta^{1-\beta} & \text { if } \beta \neq 1, \\
\omega_{\mu}(|x-y|)|\ln \delta| & \text { if } \beta=1,\end{cases}
\end{aligned}
$$

with, if $\beta=1, \omega_{\mu}(\cdot)$ such that $\omega_{\mu}(r)|\ln r| \rightarrow 0$ as $r \rightarrow 0$. Suppose also that the nonlinearity $F$ satisfies $(\mathrm{H})$ for some parameters $k, \tau, \theta$.

(i) If

$$
\theta>\frac{1}{2}(2-\beta) \quad \text { and } \quad \begin{cases}k=\beta & \text { if } \beta>1, \\ k<\beta & \text { if } \beta \leq 1,\end{cases}
$$

then any bounded continuous viscosity solution $u$ of 1.1 is locally $\alpha$-Hölder continuous for $\alpha$ small enough. More precisely, $\alpha$ must satisfy: $\alpha<1$ if $\beta \geq 1$ and $\alpha<(\beta-k) /(1-k)$ if $\beta<1$.

(ii) If $\beta<1$ and if we assume moreover that $C_{F}=0$ in $(\mathrm{H})$ and $\tau>k\left(\beta^{-1}-1\right)$, then $u$ is $\beta$-Hölder continuous. 
Moreover, in both cases (i) and (ii), the $C^{0, \alpha}$ and $C^{0, \beta}$ estimates depend on $\|u\|_{\infty}, N$ (dimension), the constants $\tilde{C}_{\mu}, C_{\mu}(\eta)$ and the function $\omega_{\mu}$ appearing in 3.2)-3.4, and on the constants and functions appearing in $(\mathrm{H})$.

We now turn to Lévy-Itô operators.

Theorem 3.2 (Hölder continuity for Lévy-Itô operators). Assume that the function $j$ appearing in the definition of $\mathcal{I}_{L I}$ satisfies: there exist $c_{0}, C_{0}>$ such that, for any $x \in \Omega$ and $z \in \mathbb{R}^{N}$,

$$
c_{0}|z| \leq|j(x, z)| \leq C_{0}|z|, \quad|j(x, z)-j(y, z)| \leq C_{0}|z||x-y|^{\tilde{\theta}}
$$

with some $\tilde{\theta} \in(0,1)$. Assume, in addition, that the measure $\mu$ satisfies: there exist $\beta \in$ $(0,2)$, a constant $\tilde{C}_{\mu}>0$ and, for any $\eta \in(0,1)$, a constant $C_{\mu}(\eta)>0$ such that for any $x \in \Omega, d \in \mathcal{S}^{N-1}$, and $\eta, \delta \in(0,1)$,

$$
\int_{B}|j(x, z)|^{2} \mu(d z)+\int_{\mathbb{R}^{N} \backslash B} \mu(d z) \leq \tilde{C}_{\mu}, \quad \int_{\mathcal{C}_{\delta, \eta}(x)}|j(x, z)|^{2} \mu(d z) \geq C_{\mu}(\eta) \delta^{2-\beta}
$$

where $\mathcal{C}_{\delta, \eta}(x):=\{z:|j(x, z)| \leq \delta,|d \cdot j(x, z)| \geq(1-\eta)|j(x, z)|\}$, and that, moreover, for $\delta$ small enough,

$$
\int_{B \backslash B_{\delta}}|z| \mu(d z) \leq \begin{cases}\tilde{C}_{\mu} \delta^{1-\beta} & \text { if } \beta \neq 1, \\ \tilde{C}_{\mu}|\ln \delta| & \text { if } \beta=1 .\end{cases}
$$

Assume, finally, that the non-linearity $F$ satisfies $(\mathrm{H})$ with parameters $k, \tau, \theta$. If

$$
\theta, \tilde{\theta}>\frac{1}{2}(2-\beta) \quad \text { and } \quad \begin{cases}k=\beta & \text { if } \beta>1 \\ k<\beta & \text { if } \beta \leq 1\end{cases}
$$

then any bounded continuous viscosity solution $u$ of $[1.1)$ with $\mathcal{I}[x, u]$ replaced with (1.4) is locally $\alpha$-Hölder continuous for any $\alpha<\min (1, \beta)$.

If, in addition, $C_{F}=0$ in $(\mathrm{H})$ and $\tau>k(1-\beta) \beta^{-1}$, then $u$ is $\beta$-Hölder continuous when $\beta<1$.

Moreover, the $C^{0, \alpha}$ estimate depends on $\|u\|_{\infty}, N$ and the constants and functions appearing in $(\mathrm{H})$ and $3.5-3.7$.

Remark 3.3. It is worth pointing out that (3.5)-3.7) are analogues of 3.2)-3.4 but they do not imply them. It is easy to see that the first condition of 3.5 together with (3.6) implies (3.2) but 3.3) and (3.4) do not derive from the second condition of 3.5) and 3.7.

Typically we have in mind the measures $\mu$ which satisfy, for some $C_{\mu}^{ \pm}>0$ and $\beta \in(0,2)$,

$$
\frac{C_{\mu}^{-}}{|z|^{N+\beta}} d z \leq \mu(d z) \leq \frac{C_{\mu}^{+}}{|z|^{N+\beta}} d z .
$$


and functions $j(x, z)$ such that $z \mapsto j(x, z)$ has an inverse function $J(x, Z)$ and there exist $c_{0}, C_{0}>0$ such that

$$
\left\{\begin{aligned}
\forall(x, z) \in B_{r}\left(x_{0}, 0\right), & c_{0}|z| \leq|j(x, z)| \leq C_{0}|z|, \\
\forall(x, Z) \in B_{R}\left(x_{0}, 0\right), & c_{0}|Z| \leq|j(x, Z)| \leq C_{0}|Z|, \\
\forall Z \in \mathbb{R}^{N}, & c_{0} \leq\left|\operatorname{det} D_{z} J\left(x_{0}, Z\right)\right|, \\
\forall z \in \mathbb{R}^{N}, & |j(x, z)-j(y, z)| \leq C_{0}|z||x-y|^{\tilde{\theta}},
\end{aligned}\right.
$$

and these are the properties we will use. We are in such a case if, for instance, for any $x$, $D_{z} j(x, z)$ exists for $|z|$ small enough, is continuous in $(x, z)$ and non-singular for $z=0$. Such a condition appears in [6].

\section{Comments and examples}

In this section, we comment on the assumptions of the main theorems and give examples of applications. More precisely, we illustrate the different terms appearing in the structure condition $(\mathrm{H})$; we give examples of non-local operators of type (1.2) and (1.4); eventually, we give a regularity result that applies to the Bellman-Isaacs equation.

\subsection{Non-linearities}

In this subsection, we illustrate the structure condition $(\mathrm{H})$ we used in Theorems 3.1 and 3.2 and, to do so, we consider the model equation

$$
-\operatorname{Tr}\left(A(x) D^{2} u\right)-c(x) \mathcal{I}[x, u]+H(x, u, D u)=0 \quad \text { in } \Omega,
$$

where $A: \Omega \rightarrow \mathbb{S}^{N}, c: \Omega \rightarrow \mathbb{R}$ and $H: \Omega \times \mathbb{R} \times \mathbb{R}^{N} \rightarrow \mathbb{R}$ are continuous functions and $\mathcal{I}[x, u]$ is a non-local term of type $(1.2)$ or 1.4 .

First, the equation (4.1) has to be degenerate elliptic and therefore we assume that for all $x \in \Omega, A(x) \geq 0$ and $c(x) \geq 0$. For $A$, we are even going to use the more restrictive assumption (but natural in the probabilistic framework)

$$
\text { for all } x \in \Omega, \quad A(x)=\sigma(x) \sigma^{*}(x),
$$

where $\sigma$ is a continuous function which maps $\Omega$ into the space of $N \times p$ matrices for some $p \leq N$.

We go back to the structure condition $(\mathrm{H})$. It combines two different terms. The first one permits handling equations that are strictly elliptic in the usual sense. The second permits one to handle non-local equations that are strictly elliptic in a generalized (nonlocal) sense. Notice that imposing $\Lambda_{1}(x)+\Lambda_{2}(x) \geq \Lambda_{0}>0$ means that, at each point $x \in \Omega$, the non-linearity is either strictly elliptic in the classical (non-linear) sense or strictly elliptic in the generalized (non-local) sense.

A typical situation is the following: we are given two open subsets $\mathcal{O}_{1}, \mathcal{O}_{2}$ of $\Omega$ such that $\mathcal{O}_{1} \cup \mathcal{O}_{2}=\Omega$ and the closure of $\left(\mathcal{O}_{1}\right)^{c}$ is included in $\mathcal{O}_{2}$; moreover we know that $F$ satisfies $(\mathrm{H})$ in $\mathcal{O}_{1}$ with $\Lambda_{1}(x) \equiv 1, \Lambda_{2}(x) \equiv 0$, while $F$ satisfies $(\mathrm{H})$ in $\mathcal{O}_{2}$ with 
$\Lambda_{1}(x) \equiv 0, \Lambda_{2}(x) \equiv 1$. Then, if $\Lambda$ is a continuous function in $\Omega$ which equals 1 on the closure of $\left(\mathcal{O}_{1}\right)^{c}$ and has support in $\mathcal{O}_{2}$, it is easy to check that $F$ satisfies (H) with $\Lambda_{1}(x) \equiv 1-\Lambda(x), \Lambda_{2}(x) \equiv \Lambda(x)$.

For equation 4.1], the structure condition $(\mathrm{H})$ means that we assume

$$
A(x) \geq \Lambda_{1}(x) I \quad \text { and } \quad c(x) \geq \Lambda_{2}(x) \quad \text { in } \Omega .
$$

Typically this means that the second-order operator $-\operatorname{Tr}\left(A(x) D^{2} u\right)$ is uniformly elliptic in $\mathcal{O}_{1}$, while there is no degeneracy in the non-local variable $l$ in $\mathcal{O}_{2}$. Of course, in the conditions " $\Lambda_{1}(x) \equiv 1$ in $\mathcal{O}_{1}$ " or " $\Lambda_{2}(x) \equiv 1$ in $\mathcal{O}_{2}$ ", the " $\equiv 1$ " may be replaced by “” $\equiv$ " with $\Lambda>0$.

Besides this ellipticity assumption, we have to assume that $A$ (or more precisely $\sigma$ ) satisfies suitable continuity assumptions in $\mathcal{O}_{1}$ and $\mathcal{O}_{2}$ : this appears in $(\mathrm{H})$ in the second subterms of the $\Lambda_{1}, \Lambda_{2}$-terms. To describe these assumptions, we recall a standard computation which appeared for the first time in Ishii [12]: we assume that $\sigma$ is bounded and uniformly continuous in $\Omega$ and we denote by $\omega_{\sigma}$ its modulus of continuity. We apply (3.1) to the vector $z=\left(z_{1}, z_{2}\right)$ with $z_{1}=\sigma(\bar{x}) e, z_{2}=\sigma(\bar{y}) e$ and an arbitrary $e \in \mathcal{S}^{N-1}$, and get

$$
\sigma^{T}(\bar{x}) X \sigma(\bar{x}) e \cdot e-\sigma^{T}(\bar{y}) Y \sigma(\bar{y}) e \cdot e \leq \frac{1}{\bar{\varepsilon}} \omega_{\sigma}^{2}(|\bar{x}-\bar{y}|)+L\|\sigma\|_{\infty}^{2}
$$

(we used that $Z \leq I$ ). Therefore

$$
\operatorname{Tr}(A(\bar{x}) X)-\operatorname{Tr}(A(\bar{y}) Y) \leq \frac{1}{\bar{\varepsilon}} d \omega_{\sigma}^{2}(|\bar{x}-\bar{y}|)+L d\|\sigma\|_{\infty}^{2} .
$$

Hence, choose $\omega_{F}(r)=d \omega_{\sigma}^{2}(r)$ and $\chi(L, \eta)=L d\|\sigma\|_{\infty}^{2}$. It is worth pointing out that, in the uniformly elliptic case (i.e. in $\mathcal{O}_{1}$ ), $A$ or $\sigma$ is only required to be continuous while, in $\mathcal{O}_{2}, \sigma$ has to be Hölder continuous (the same computations as above provide the $|x-y|^{2 \theta}$ term if $\left.\omega_{\sigma}(r)=C r^{\theta}\right)$ in order to take advantage of the ellipticity of the non-local term.

We now turn to the non-local term. Our main remark is the following: in our formulation, the non-local term $l$ is in fact $c(x) \mathcal{I}[x, u]$ and not only $\mathcal{I}[x, u]$. In that way, assumption $(\mathrm{H})$ is obviously satisfied by $(4.1)$. On the other hand, in order to verify the assumptions of Theorem 3.1. one has to replace the measure $\mu_{x}(d z)$ with $\tilde{\mu}_{x}(d z):=c(x) \mu_{x}(d z)$ and check if the conditions are satisfied by this new measure. In other words, in the case of $[1.2]$, we rewrite $c(x) \mathcal{I}[x, u]$ as

$$
\int_{\mathbb{R}^{N}}\left(u(x+z)-u(x)-D u(x) \cdot z \mathbf{1}_{B}(z)\right) \tilde{\mu}_{x}(d z) .
$$

Therefore, the continuity assumptions on $\mu_{x}$ in Theorem 3.1 are indeed continuity assumptions on $\tilde{\mu}_{x}$ and, for this reason, they contain continuity assumptions on both $\mu_{x}$ and $c$.

A priori a similar approach could be used for 1.4 but this really means that we use Theorem 3.1 instead of Theorem 3.2 in this case.

It remains to consider the first order term $H(x, u, D u)$ in 4.1 : this appears in $(\mathrm{H})$ in the third, fourth and (part of the) fifth subterms of $\Lambda_{1}, \Lambda_{2}$-terms. As is classical for (local) 
equations, we have to require growth conditions with respect to $D u$ : quadratic growth $\left(|p|^{2}\right)$ when the equation is uniformly elliptic, and a $|p|^{k}$ growth with $k$ depending on the measure when the strong ellipticity comes from the non-local term.

For example, in the case of the fractional Laplacian $(-\Delta)^{\beta / 2}$, the natural growth turns out to be $k=\beta$, even if Theorem 3.1 shows that the case $\beta \leq 1$ is a little more particular. We refer the reader to Subsection 4.3 where an example of the equation involving a fractional Laplacian is treated in detail.

These growth conditions on the gradient have to be combined with the regularity of coefficients: we are able to treat gradient terms of the form $c(x)|D u(x)|^{m}$ with $m=2$ if $c$ is merely bounded, and $m=2+\tau$ if $c$ is locally $\tau$-Hölder continuous. We leave details to the reader.

We say more about these assumptions in the next subsections. In particular, we treat equations that are not exactly of the form (1.1) but can be handled with the same techniques (see Subsection 4.4).

\subsection{Singular measures}

The model singular measure is the Lévy measure associated with the fractional Laplacian $(-\Delta)^{\beta / 2}$. In this case, $d \mu_{x}(z)=d \mu(z)=d z /|z|^{N+\beta}$ with $0<\beta<2$.

A second simple example of a measure $\mu_{x}$ is $c(x, z) \mu(d z)$ with a Lévy measure $\mu$ satisfying (3.6-3.7) with $j(x, z)=z$ and $c(x, z)$ satisfying, for any $x, y \in \Omega$ and $z \in B$,

$$
|c(x, z)-c(y, z)| \leq \omega(|x-y|) \quad \text { where } \omega(t) \rightarrow 0 \text { when } t \downarrow 0,
$$

and, for any $x \in \Omega, z \in \mathbb{R}^{N}, 0<\underline{c} \leq c(x, z) \leq \bar{c}$ for some constants $\underline{c}, \bar{c}$. One can thus easily check 3.2 -3.4 and Theorem 3.1 applies for suitable non-linearities $F$. Lévy measures associated with tempered stable Lévy processes satisfy (3.6-3.7). Indeed, in this case

$$
\mu(d z)=\mathbf{1}_{(0,+\infty)}(z)\left(G^{+} e^{-\lambda^{+}|z|} \frac{d z}{|z|^{N+\alpha}}\right)+\mathbf{1}_{(-\infty, 0)}(z)\left(G^{-} e^{-\lambda^{-}|z|} \frac{d z}{|z|^{N+\alpha}}\right) .
$$

These measures appear in financial modeling (see for instance [9]).

\subsection{A non-local equation involving the fractional Laplacian}

In order to further illustrate our results, we next consider the model equation

$$
(-\Delta)^{\beta / 2} u+b(x)|D u|^{k+\tau}+|D u|^{r}=0
$$

where $b \in C^{0, \tau}, 0<\tau<1,0<k, r<2$. In this case, condition (H) is satisfied with $\Lambda_{1}=0, \Lambda_{2}(x)=1>0, \theta>0$ arbitrary and $\tau, k, r$ as in the equation. It is easy to 
check that (3.2) is satisfied with exponent $\beta$ : first, it is a Lévy measure and if $\mathcal{C}_{\delta, \eta}$ denotes $\{|z|<|\delta|, d \cdot z \geq(1-\eta)|z|\}$, then by homogeneity and symmetry of $\mu$,

$$
\begin{aligned}
\int_{\mathcal{C}_{\delta, \eta}}|z|^{2} \mu(d z) & =\frac{\left|\mathcal{C}_{\delta, \eta}\right|}{\left|B_{\delta}\right|} \int_{B_{\delta}}|z|^{2} \mu(d z)=\frac{\left|\mathcal{C}_{1, \eta}\right|}{\left|B_{1}\right|} \int_{B_{\delta}}|z|^{2} \mu(d z) \\
& =\frac{\left|\mathcal{C}_{1, \eta}\right|}{\left|B_{1}\right|} \int_{B_{\delta}}|z|^{2-\beta-N} d z=C_{\mu}(\eta) \delta^{2-\beta} .
\end{aligned}
$$

Moreover, the other hypotheses we make on $\mu$ (namely (3.3) and (3.4)) are automatically satisfied since $\mu$ is independent of $x$ (in other words, one can choose $\omega_{\mu}=0$ ). Since $\beta<2$, we cannot allow here a quadratic growth for the gradient term; indeed, Theorems 3.1 and 3.2 work only for $k, r \leq \beta$ in the absence of local ellipticity. It is also worth pointing out that, if $r \leq 1$, we have $|p|^{r}-|p+q|^{r} \leq|q|^{r} \leq R^{r}$ and therefore, even if $\beta<1$, any such $r$ works since the $R^{r}$ can be absorbed in the $\chi(L, \eta)$-term.

\subsection{The Bellman-Isaacs equation}

Let us illustrate Theorem 3.2 by considering an important second-order non-linear elliptic integro-differential equations appearing in the study of stochastic control of processes with jumps, namely the Bellman-Isaacs equation. Let us mention the work of Jakobsen and Karlsen [14] in the evolution case where the authors use completely different techniques.

Corollary 4.1. Consider the following Bellman-Isaacs equation in $\mathbb{R}^{N}$ :

$c u+\sup _{\lambda \in \Lambda} \inf _{\gamma \in \Gamma}\left\{-\frac{1}{2} \operatorname{Tr}\left(\sigma_{\lambda, \gamma}(x) \sigma_{\lambda, \gamma}^{*}(x) D^{2} u\right)-b_{\lambda, \gamma}(x) \cdot D u-\mathcal{I}_{L I}^{\lambda, \gamma}[x, u]-f_{\lambda, \gamma}(x)\right\}=0$

with $c \geq 0$ and where $\mathcal{I}_{L I}^{\lambda, \gamma}[x, u]$ is a family of Lévy-Itô operators associated with a common Lévy measure $\mu$ and a family of functions $j_{\lambda, \gamma}(x, z)$. Assume that

1. $\mu$ satisfies 3.6-3.7) with constants independent of $\lambda, \mu$,

2. there exist $c_{0}, C_{0}>0$ and $\tilde{\theta} \in(0,1)$ such that for any $(\lambda, \gamma) \in \Lambda \times \Gamma, j_{\lambda, \gamma}$ satisfies (3.5),

3. $\sigma_{\lambda, \gamma}, b_{\lambda, \gamma}$ and $f_{\lambda, \gamma}$ satisfy, for some $\theta \in(0,1)$ (and some constant $C_{F}>0$ ),

$$
\forall \alpha, \beta, \quad\left\|\sigma_{\lambda, \gamma}\right\|_{0, \theta}+\left\|b_{\lambda, \gamma}\right\|_{0, \theta}+\left\|f_{\lambda, \gamma}\right\|_{0, \theta} \leq C_{F} .
$$

If $\theta, \tilde{\theta}>\frac{1}{2}(2-\beta)$, then any bounded viscosity solution u of 4 4.2] is $\alpha$-Hölder continuous for any $\alpha<1$ if $\beta \geq 1$, and for $\alpha<(\beta-k) /(1-k)$ if $\beta<1$.

Proof. Note that (4.2) is not exactly of the form 1.1. Nevertheless, the proof of Theorem 3.2 we present below can be adapted to this framework. It is enough to check that the structure condition $(\mathrm{H})$ is satisfied by the linear equations

$$
F_{\lambda, \gamma}(x, u, p, A, l)=c u-\frac{1}{2} \operatorname{Tr}\left(\sigma_{\lambda, \gamma}(x) \sigma_{\lambda, \gamma}^{*}(x) A\right)-b_{\lambda, \gamma}(x) \cdot p-l_{\lambda, \gamma}-f_{\lambda, \gamma}(x),
$$

with constants and functions appearing in $(\mathrm{H})$ independent of $\lambda, \gamma$. 
Indeed, if this is the case, then we just have to use the standard inequality

$$
\sup _{\lambda} \inf _{\gamma}(\cdots)-\sup _{\lambda} \inf _{\gamma}(\cdots) \leq \sup _{\lambda, \gamma}(\cdots-\cdots),
$$

and we can conclude in this more general case too.

\section{Proofs of Theorems 3.1 and 3.2}

We prove successively Theorems 3.1 and 3.2 On one hand, the proofs are very similar and we will skip details in the proof of the second theorem when adapting arguments used in the proof of the first one. On the other hand, we need to use every parameter very precisely. This is the reason why constants are computed from line to line and explicit formulae are given for each of them in order to use them later.

\subsection{Proof of Theorem 3.1}

We first prove (i). Without loss of generality, we assume that $C_{F}=1$ in the ellipticitygrowth condition $(\mathrm{H})$. In order to prove the local Hölder continuity of $u$, we are going to show that, for any $x_{0} \in \Omega$, there exists $L_{2}=L_{2}\left(x_{0}\right)$ such that, for some well chosen $\alpha \in(0,1)$ and for $L_{1}=L_{1}\left(x_{0}\right)>0$ large enough, we have

$$
M=\sup _{x, y \in \mathbb{R}^{N}}\{u(x)-u(y)-\phi(x-y)-\Gamma(x)\} \leq 0
$$

where $\phi(z)=L_{1}|z|^{\alpha}$ and $\Gamma(x)=L_{2}\left|x-x_{0}\right|^{2}$. We point out that the role of the term $\Gamma(x)$ is to localize around $x_{0}$, while the term $\phi(x-y)$ is concerned with the Hölder continuity. Proving such a result with a suitable control on $\alpha, L_{1}, L_{2}$ clearly implies the desired property.

If $\Omega \neq \mathbb{R}^{N}$, we first choose $L_{2}$ so that $u(x)-u(y)-\phi(x-y)-\Gamma(x) \leq 0$ if $x \notin \Omega$ : to do so, we first choose

$$
L_{2} \geq \frac{8\|u\|_{\infty}}{\left[d\left(x_{0}, \partial \Omega\right)\right]^{2}}
$$

If $\Omega=\mathbb{R}^{N}, L_{2}$ is arbitrary. Then we argue by contradiction: we assume that $M>0$ and we are going to get a contradiction for $L_{1}$ large enough and for a suitable choice of $\alpha$.

If the supremum defining $M$ is attained at $(\bar{x}, \bar{y})$, we deduce from $M>0$ that $\bar{x} \neq \bar{y}$ and

$$
\begin{aligned}
& |\bar{x}-\bar{y}| \leq\left(2\|u\|_{\infty} / L_{1}\right)^{1 / \alpha}=: A, \\
& \left|\bar{x}-x_{0}\right|<\sqrt{2\|u\|_{\infty} / L_{2}}=: R_{2} \leq d\left(x_{0}, \partial \Omega\right) / 2, \\
& u(\bar{x})>u(\bar{y}) .
\end{aligned}
$$

If $L_{1}$ is so large that $A<d\left(x_{0}, \partial \Omega\right) / 2$, then we have $\bar{x}, \bar{y} \in \Omega$.

Next, we pick some $\nu_{0} \in(0,1)$ and we define

$$
a=\bar{x}-\bar{y}, \quad \varepsilon=|a|, \quad \hat{a}=a /|a|, \quad \delta=v_{0} \varepsilon<\varepsilon .
$$

First, $v_{0}$ will be chosen small enough but fixed (independent of $L_{1}$ and $\varepsilon$ ). 
From the study of the maximum point property for $(\bar{x}, \bar{y})$, we also get

$$
L_{1} \varepsilon^{\alpha} \leq u(\bar{x})-u(\bar{y}) \leq \omega_{u}(\varepsilon)
$$

where $\omega_{u}$ denotes the modulus of continuity of $u$ on $B\left(x_{0}, d\left(x_{0}, \partial \Omega\right) / 2\right)$. We will use this piece of information below (see Step 4). Notice also that if $\chi(x, y)$ denotes $\phi(x-y)+$ $\Gamma(x)$, then $x \mapsto u(x)-\chi(x, \bar{y})$ (resp. $y \mapsto u(y)+\chi(\bar{x}, y)$ ) attains a global maximum (resp. minimum) at $\bar{x}$ (resp. $\bar{y}$ ) with $\chi(\cdot, \bar{y})$ (resp. $-\chi(\bar{x}, y))$ of class $C^{2}$ on $B(\bar{x}, \delta)$. In particular, we can use $\chi(\bar{x}, \cdot)$ and $-\chi(\cdot, \bar{y})$ as test functions in Definition 2.1 with any $\delta^{\prime}<\delta$.

The remainder of the proof is divided into four steps. We write down the viscosity inequalities and combine them (Step 1), we get suitable matrix inequalities from nonlocal Jensen-Ishii's lemma (Step 2), we estimate from above the difference of the nonlocal terms (Step 3) and we conclude (Step 4).

Step 1: Writing down viscosity inequalities. Let $p$ denote $D \phi(a)$ and $q$ denote $D \Gamma(\bar{x})$. We use Corollary 1 of [4] which, for $\iota>0$ small enough, provides us with two matrices $X_{\iota}, Y_{\iota} \in \mathbb{S}^{N}$ such that, for any $\delta^{\prime} \ll 1$,

$$
\begin{array}{r}
F\left(\bar{x}, u(\bar{x}), p+q, X_{\iota}, \mathcal{I}^{1, \delta^{\prime}}\left[\bar{x}, p+q, \chi_{\iota}(\cdot, \bar{y})\right]+\mathcal{I}^{2, \delta^{\prime}}[\bar{x}, p+q, u]+o_{\iota}(1)\right) \leq 0, \\
F\left(\bar{y}, u(\bar{y}), p, Y_{\iota}, \mathcal{I}^{1, \delta^{\prime}}\left[\bar{y}, p,-\chi_{\iota}(\bar{x}, \cdot)\right]+\mathcal{I}^{2, \delta^{\prime}}[\bar{y}, p, u]+o_{\iota}(1)\right) \geq 0
\end{array}
$$

(where $\chi_{\iota}$ is an approximation of $\chi$ by a localized inf-convolution, see [4]), together with the following matrix inequality:

$$
-\frac{1}{\iota} I \leq\left[\begin{array}{rr}
X_{\iota} & 0 \\
0 & -Y_{\iota}
\end{array}\right] \leq\left[\begin{array}{rr}
Z & -Z \\
-Z & Z
\end{array}\right]+2 L_{2}\left[\begin{array}{ll}
I & 0 \\
0 & 0
\end{array}\right]+o_{\iota}(1),
$$

where $Z=D^{2} \phi(a)$.

Our aim is to first let $\iota$ tend to 0 in order to get rid of all the artificial $\iota$ dependences in these inequalities; in order to do so, but also in order to apply $(\mathrm{H})$ which requires a twoside bound on the matrices, we are first going to build matrices $X, Y$ such that the above viscosity inequalities still hold if we replace $X_{\iota}, Y_{\iota}$ by $X, Y$ and such that the matrices $X, Y$ satisfy the required inequality in $(\mathrm{H})$.

Then, if we set

$$
\begin{aligned}
& l_{1}:=\mathcal{I}^{1, \delta^{\prime}}\left[\bar{x}, p+q, \chi_{\iota}(\cdot, \bar{y})\right]+\mathcal{I}^{2, \delta^{\prime}}[\bar{x}, p+q, u], \\
& l_{2}:=\mathcal{I}^{1, \delta^{\prime}}\left[\bar{y}, p,-\chi_{\iota}(\bar{x}, \cdot)\right]+\mathcal{I}^{2, \delta^{\prime}}[\bar{y}, p, u],
\end{aligned}
$$

and if we subtract the viscosity inequalities, dropping all the $\iota$ dependences, we will have

$$
0 \leq F\left(\bar{y}, u(\bar{y}), p, Y, l_{2}\right)-F\left(\bar{x}, u(\bar{x}), p+q, X, l_{1}\right) .
$$

In order to get the desired contradiction, in the rest of the proof we obtain various estimates, in particular on the differences $X-Y$ and $\mathcal{I}^{2, \delta^{\prime}}[\bar{x}, p+q, u]-\mathcal{I}^{2, \delta^{\prime}}[\bar{y}, p, u]$, in order to apply the ellipticity-growth condition $(\mathrm{H})$ to show that the right-hand side of this 
inequality is strictly negative. We point out that, since we are going to let first $\delta^{\prime}$ tend to 0 , the terms $\mathcal{I}^{1, \delta^{\prime}}\left[\bar{x}, p+q, \chi_{\iota}(\cdot, \bar{y})\right], \mathcal{I}^{1, \delta^{\prime}}\left[\bar{y}, p,-\chi_{\iota}(\bar{x}, \cdot)\right]$ create no difficulty because they tend to 0 with $\delta^{\prime}$.

Step 2: Building and estimating the matrices $X, Y$. We follow here ideas introduced by Crandall and Ishii [11] to obtain these matrices, by using only the upper bounds on $X_{\iota}, Y_{\iota}$. We rewrite 5.3 as: for any $z_{1}, z_{2} \in \mathbb{R}^{N}$,

$$
X_{\iota} z_{1} \cdot z_{1}-Y_{\iota} z_{2} \cdot z_{2} \leq Z\left(z_{1}-z_{2}\right) \cdot\left(z_{1}-z_{2}\right)+2 L_{2}\left|z_{1}\right|^{2} .
$$

We have dropped the $o_{t}(1)$-term on the right-hand side for the sake of simplicity since it plays no role. In fact, we use the previous matrix inequality in the form

$$
\left(X_{\iota}-2 L_{2} I\right) z_{1} \cdot z_{1}-Y_{\iota} z_{2} \cdot z_{2} \leq Z\left(z_{1}-z_{2}\right) \cdot\left(z_{1}-z_{2}\right) .
$$

Next we have to compute $Z$, as well as, for the rest of the proof, the derivatives of $\phi$. It will be convenient to do the proof for $\phi(x)=\varphi(|x|)$ for a general smooth function $\varphi: \mathbb{R}^{+} \rightarrow \mathbb{R}$. We thus get, for any $b \in \mathbb{R}^{N}$,

$$
D \phi(b)=\varphi^{\prime}(|b|) \hat{b}, \quad D^{2} \phi(b)=\frac{\varphi^{\prime}(|b|)}{|b|} P_{b^{\perp}}+\varphi^{\prime \prime}(|b|) \hat{b} \otimes \hat{b}
$$

where $\hat{b}=b /|b|$ and $P_{b^{\perp}}=I-\hat{b} \otimes \hat{b}$ is the projection on the orthogonal complement of $b$. Hence, if $\varphi(r)=L_{1} r^{\alpha}$, we get

$$
\begin{aligned}
D \phi(b) & =L_{1} \alpha|b|^{\alpha-2} b, \\
D^{2} \phi(b) & =L_{1}\left(\alpha|b|^{\alpha-2} I+\alpha(\alpha-2)|b|^{\alpha-4} b \otimes b\right) \\
& =L_{1} \alpha|b|^{\alpha-4}\left(|b|^{2} I-(2-\alpha) b \otimes b\right), \\
D^{2} \phi(b) & \leq L_{1} \alpha|b|^{\alpha-2} I .
\end{aligned}
$$

We have

$$
Z=\frac{1}{\bar{\varepsilon}}(I-(2-\alpha) \hat{a} \otimes \hat{a}) \quad \text { with } \quad \bar{\varepsilon}=\left(L_{1} \alpha \varepsilon^{\alpha-2}\right)^{-1} .
$$

Now we go back to the $X_{\iota}, Y_{\iota}$ inequality; we apply to it a sup-convolution in both variables $z_{1}$ and $z_{2}$ with parameter $\frac{1}{4} \bar{\varepsilon}$. Noticing that this corresponds to an inf-convolution on the $\left(Y_{l} z_{2} \cdot z_{2}\right)$-term, we easily get, with the notation introduced at the end of the Introduction,

$$
\left(X_{\iota}-2 L_{2} I\right)^{\bar{\varepsilon} / 4} z_{1} \cdot z_{1}-\left(Y_{l}\right) \bar{\varepsilon} / 4 z_{2} \cdot z_{2} \leq Z^{\bar{\varepsilon} / 2}\left(z_{1}-z_{2}\right) \cdot\left(z_{1}-z_{2}\right) .
$$

On the other hand, tedious but easy, explicit computations (which are provided in the Appendix) yield

$$
Z^{\bar{\varepsilon} / 2}=2 \bar{\varepsilon}^{-1}(I-(1+\varpi) \hat{a} \otimes \hat{a}) \quad \text { with } \quad \varpi:=\frac{1-\alpha}{3-\alpha}>0 .
$$

Notice that $0<\varpi<1 / 3$. 
If we set $X=\left(X_{\iota}-2 L_{2} I\right)^{\bar{\varepsilon} / 4}+2 L_{2} I, Y=\left(Y_{\iota}\right) \bar{\varepsilon} / 4$, then $X, Y$ satisfy (3.1) with $L=2 L_{2}$, and since $X_{\iota} \leq X$ and $Y \leq Y_{\iota}$, the viscosity inequalities still hold for $X$ and $Y$ because $F$ is degenerate elliptic.

From this new form of inequality 5.3 , we can obtain several types of estimates on $X$ and $Y$. First, choosing $z_{2}=-z_{1}=\hat{a}$, we get

$$
X \hat{a} \cdot \hat{a}-Y \hat{a} \cdot \hat{a} \leq-8 \varpi / \bar{\varepsilon}+O\left(L_{2}\right)=-8 L_{1} \alpha \varpi \varepsilon^{\alpha-2}+O\left(L_{2}\right) .
$$

Next choosing $z_{2}=z_{1}=z$ with $z$ being orthogonal to $\hat{a}$, we have

$$
X z \cdot z-Y z \cdot z \leq O\left(L_{2}\right)
$$

In particular, this yields

$$
\operatorname{Tr}(X-Y) \leq-8 L_{1} \alpha \varpi \varepsilon^{\alpha-2}+O\left(L_{2}\right) .
$$

Step 3: Estimates of the non-local terms. The difference of the non-local terms, denoted by $T_{n l}$, can be rewritten as follows (we recall that $B=B(0,1)$ ):

$$
\begin{aligned}
T_{n l}= & \int_{|z| \geq \delta^{\prime}}\left[u(\bar{x}+z)-u(\bar{x})-(p+q) \cdot z \mathbf{1}_{B}(z)\right] \mu_{\bar{x}}(d z) \\
& -\int_{|z| \geq \delta^{\prime}}\left[u(\bar{y}+z)-u(\bar{y})-p \cdot z \mathbf{1}_{B}(z)\right] \mu_{\bar{y}}(d z) .
\end{aligned}
$$

In order to estimate it, we split the domain of integration $\left\{|z| \geq \delta^{\prime}\right\}$ into three pieces: $\mathbb{R}^{N} \backslash B$ which leads to the $T_{1}$ term below; $\mathcal{C}=\left\{z \in B_{\delta}:|z \cdot \hat{a}| \geq(1-\eta)|z|\right\} \subset B$ which leads to the $T_{2}$ term below; and $B \backslash \mathcal{C}$ which leads to the $T_{3}$ term below.

In order not to add further technicalities, we assume from now on that $\delta^{\prime}=0$; the reader can check that if $\delta^{\prime}>0$, the estimates we present below remain valid up to $o_{\delta^{\prime}}(1)$.

Therefore we have to estimate from above $T_{n l}=T_{1}+T_{2}+T_{3}+o_{\delta^{\prime}}(1)$ with

$$
\begin{aligned}
T_{1}= & \int_{|z| \geq 1}[u(\bar{x}+z)-u(\bar{x})] \mu_{\bar{x}}(d z)-\int_{|z| \geq 1}[u(\bar{y}+z)-u(\bar{y})] \mu_{\bar{y}}(d z) \\
T_{2}= & \int_{\mathcal{C}}[u(\bar{x}+z)-u(\bar{x})-(p+q) \cdot z] \mu_{\bar{x}}(d z)-\int_{\mathcal{C}}[u(\bar{y}+z)-u(\bar{y})-p \cdot z] \mu_{\bar{y}}(d z) \\
T_{3}= & \int_{B \backslash \mathcal{C}}[u(\bar{x}+z)-u(\bar{x})-(p+q) \cdot z] \mu_{\bar{x}}(d z) \\
& -\int_{B \backslash \mathcal{C}}[u(\bar{y}+z)-u(\bar{y})-p \cdot z] \mu_{\bar{y}}(d z) .
\end{aligned}
$$

For the reader's convenience, we recall that $p=D \phi(a)$ and $q=D \Gamma(\bar{x})$.

Estimate of $T_{1}$. Since $u$ is bounded and so are the measures $\mu_{x}$ away from the origin, we conclude that $T_{1}$ is bounded, uniformly with respect to all the parameters we introduced. More precisely,

$$
T_{1} \leq C_{1}
$$


where

$$
C_{1}=4\|u\|_{\infty} \sup _{x \in B\left(x_{0}, d\left(x_{0}, \partial \Omega\right) / 2\right)} \mu_{x}\left(\mathbb{R}^{N} \backslash B\right) .
$$

Estimate of $T_{2}$. We estimate $T_{2}$ from above by using the definition of $M$. Indeed, its definition provides the following key inequality:

$$
\begin{aligned}
u(\bar{x}+d)-u(\bar{x})-(p+q) \cdot d \leq & u\left(\bar{y}+d^{\prime}\right)-u(\bar{y})-p \cdot d^{\prime} \\
& +\left\{\phi\left(a+d-d^{\prime}\right)-\phi(a)-D \phi(a) \cdot\left(d-d^{\prime}\right)\right\} \\
& +\{\Gamma(\bar{x}+d)-\Gamma(\bar{x})-D \Gamma(\bar{x}) \cdot d\} .
\end{aligned}
$$

We then use (5.11) with $d=z$ and $d^{\prime}=0$ (resp. with $d=0$ and $d^{\prime}=z$ ). We obtain

$$
\begin{aligned}
T_{2} \leq & \int_{\mathcal{C}}[\phi(a+z)-\phi(a)-D \phi(a) \cdot z] \mu_{\bar{x}}(d z) \\
& +\int_{\mathcal{C}}[\phi(a-z)-\phi(a)+D \phi(a) \cdot z] \mu_{\bar{y}}(d z) \\
& +\int_{\mathcal{C}}[\Gamma(\bar{x}+z)-\Gamma(\bar{x})-D \Gamma(\bar{x}) \cdot z] \mu_{\bar{x}}(d z)
\end{aligned}
$$

We now use a second-order Taylor expansion in each integral. First, according to the form of $\Gamma$, we have

$$
\int_{\mathcal{C}}[\Gamma(\bar{x}+z)-\Gamma(\bar{x})-D \Gamma(\bar{x}) \cdot z] \mu_{\bar{x}}(d z)=L_{2} \int_{\mathcal{C}}|z|^{2} \mu_{\bar{x}}(d z)=O\left(L_{2}\right) .
$$

Next, for the other two terms and for a general $\phi(x)=\varphi(|x|)$, we recall [5.5] and we finally get

$$
\begin{aligned}
T_{2} \leq & \frac{1}{2} \int_{\mathcal{C}} \sup _{t \in(-1,1)}\left(\frac{\varphi^{\prime}(|a+t z|)}{|a+t z|} P_{(a+t z)^{\perp}} z \cdot z+\varphi^{\prime \prime}(|a+t z|)(\widehat{a+t z} \cdot z)^{2}\right)\left(\mu_{\bar{x}}+\mu_{\bar{y}}\right)(d z) \\
& +O\left(L_{2}\right) .
\end{aligned}
$$

and using next the equality $\varphi(r)=L_{1} r^{\alpha}$, we obtain

$$
\begin{aligned}
T_{2} \leq & \frac{L_{1} \alpha}{2} \int_{\mathcal{C}} \sup _{t \in(-1,1)}|a+t z|^{\alpha-4}\left(|a+t z|^{2}|z|^{2}-(2-\alpha)((a+t z) \cdot z)^{2}\right)\left(\mu_{\bar{x}}+\mu_{\bar{y}}\right)(d z) \\
& +O\left(L_{2}\right) .
\end{aligned}
$$

We use the notation $b$ for $a+t z$ and we estimate $|b|$ and $b \cdot z$ for $z \in \mathcal{C}$ as follows:

$$
\begin{aligned}
|b| & \leq \varepsilon+t|z| \leq \varepsilon+\delta \leq\left(1+v_{0}\right) \varepsilon, \\
|b \cdot z| & =\left.|a \cdot z+t| z\right|^{2}|\geq(1-\eta) \varepsilon| z|-\delta| z\left|\geq\left(1-\eta-v_{0}\right) \varepsilon\right| z \mid, \\
|b|^{2}|z|^{2}-(2-\alpha)(b \cdot z)^{2} & \leq\left(1+v_{0}\right)^{2} \varepsilon^{2}|z|^{2}-(2-\alpha)\left(1-\eta-v_{0}\right)^{2} \varepsilon^{2}|z|^{2} \\
& \leq\left(\left(1+v_{0}\right)^{2}-(2-\alpha)\left(1-\eta-v_{0}\right)^{2}\right) \varepsilon^{2}|z|^{2}
\end{aligned}
$$


where we choose $\eta, v_{0}$ small enough so that $1-\eta-v_{0}>0$ and

$$
(2-\alpha)\left(1-\eta-v_{0}\right)^{2}-\left(1+v_{0}\right)^{2}>0 .
$$

Hence

$$
T_{2} \leq-L_{1} C_{4} \varepsilon^{\alpha-2} \int_{\mathcal{C}}|z|^{2}\left(\mu_{\bar{x}}+\mu_{\bar{y}}\right)(d z)+O\left(L_{2}\right)
$$

with

$$
C_{4}=\frac{\alpha}{2}\left((2-\alpha)\left(1-\eta-v_{0}\right)^{2}-\left(1+v_{0}\right)^{2}\right)\left(1+v_{0}\right)^{\alpha-4} .
$$

Using (3.2), we thus obtain

$$
T_{2} \leq-L_{1} C_{4} C_{\mu}(\eta) \varepsilon^{\alpha-2} \delta^{2-\beta}+O\left(L_{2}\right)
$$

and finally,

$$
T_{2} \leq-L_{1} C_{5} \varepsilon^{\alpha-\beta}+O\left(L_{2}\right)
$$

with

$$
C_{5}=C_{4} C_{\mu}(\eta) v_{0}^{2-\beta}
$$

Estimate of $T_{3}$. In order to estimate $T_{3}$ from above, it is convenient to introduce

$$
\mathcal{U}_{1}(z)=u(\bar{x}+z)-u(\bar{x})-(p+q) \cdot z, \quad \mathcal{U}_{2}(z)=u(\bar{y}+z)-u(\bar{y})-p \cdot z,
$$

and write

$$
T_{3}=\int_{B \backslash \mathcal{C}} \mathcal{U}_{1}(z) \mu_{\bar{x}}(d z)-\int_{B \backslash \mathcal{C}} \mathcal{U}_{2}(z) \mu_{\bar{y}}(d z) .
$$

We first remark that 5.11 with, successively, $\left(d, d^{\prime}\right)=(z, z),\left(d, d^{\prime}\right)=(z, 0)$ and $\left(d, d^{\prime}\right)=(0, z)=0$ yields

$$
\left\{\begin{array}{l}
\mathcal{U}_{1}(z)-\mathcal{U}_{2}(z) \leq \Gamma(\bar{x}+z)-\Gamma(\bar{x})-D \Gamma(\bar{x}) \cdot z \\
\mathcal{U}_{1}(z) \leq(\phi(a+z)-\phi(a)-D \phi(a) \cdot z)+(\Gamma(\bar{x}+z)-\Gamma(\bar{x})-D \Gamma(\bar{x}) \cdot z) \\
\mathcal{U}_{2}(z) \geq-(\phi(a-z)-\phi(a)+D \phi(a) \cdot z)
\end{array}\right.
$$

We next consider the signed measure $\mu(d z)=\mu_{\bar{x}}(d z)-\mu_{\bar{y}}(d z)$. It can be represented by two non-negative measures $\mu^{ \pm}$using the Hahn-Jordan decomposition: we write $\mu=$ $\mu^{+}-\mu^{-}$where $\mu^{+}, \mu^{-}$are respectively the positive and negative parts of $\mu$. We would like next to introduce a measure $\min \left(\mu_{\bar{x}}, \mu_{\bar{y}}\right)$. To make it precise, we use the Hahn decomposition of $\mathbb{R}^{N}$ with respect to $\mu$ : if $K$ denotes the support of $\mu^{+}$, we define $\bar{\mu}=\mathbf{1}_{K} \mu_{\bar{y}}+\left(1-\mathbf{1}_{K}\right) \mu_{\bar{x}}$. We now rewrite $T_{3}$ with these measures. We use

$$
\mu_{\bar{x}}=\mu^{+}+\bar{\mu} \quad \text { and } \quad \mu_{\bar{y}}=\mu^{-}+\bar{\mu}
$$


together with 5.17) to get

$$
\begin{aligned}
T_{3}= & \int_{B \backslash \mathcal{C}}\left(\mathcal{U}_{1}(z)-\mathcal{U}_{2}(z)\right) \bar{\mu}(d z)+\int_{B \backslash \mathcal{C}} \mathcal{U}_{1}(z) \mu^{+}(d z)-\int_{B \backslash \mathcal{C}} \mathcal{U}_{2}(z) \mu^{-}(d z) \\
\leq & \int_{B \backslash \mathcal{C}}[\Gamma(\bar{x}+z)-\Gamma(\bar{x})-D \Gamma(\bar{x}) \cdot z] \bar{\mu}(d z) \\
& +\int_{B \backslash \mathcal{C}}[\phi(a+z)-\phi(a)-D \phi(a) \cdot z] \mu^{+}(d z) \\
& +\int_{B \backslash \mathcal{C}}[\Gamma(\bar{x}+z)-\Gamma(\bar{x})-D \Gamma(\bar{x}) \cdot z] \mu^{+}(d z) \\
& +\int_{B \backslash \mathcal{C}}[\phi(a-z)-\phi(a)+D \phi(a) \cdot z] \mu^{-}(d z) .
\end{aligned}
$$

In order to estimate the right hand side, we first remark that

$$
\int_{B \backslash \mathcal{C}}[\Gamma(\bar{x}+z)-\Gamma(\bar{x})-D \Gamma(\bar{x}) \cdot z]\left(\bar{\mu}+\mu^{+}\right)(d z) \leq L_{2} \int_{B}|z|^{2} \mu_{\bar{x}}(d z) \leq \tilde{C}_{\mu} L_{2} .
$$

Next, for the other two terms, we split the integration domain into $B \backslash B_{\delta}$ and $B_{\delta} \backslash \mathcal{C}$. On $B_{\delta} \backslash \mathcal{C}$, we use once again a second-order Taylor expansion for $\phi$ while, on $B \backslash B_{\delta}$, we use the concavity of the function $t \mapsto L_{1} t^{\alpha}$ on $(0,+\infty)$ to obtain

$$
\begin{aligned}
\phi\left(a+z^{\prime}\right)-\phi(a)-D \phi(a) & \cdot z^{\prime} \leq L_{1}\left(|a|+\left|z^{\prime}\right|\right)^{\alpha}-L_{1}|a|^{\alpha}-D \phi(a) \cdot z^{\prime} \\
& \leq \alpha L_{1}|a|^{\alpha-1}\left|z^{\prime}\right|+\left|D \phi(a) \cdot z^{\prime}\right| \leq 2 \alpha L_{1}|a|^{\alpha-1}\left|z^{\prime}\right| .
\end{aligned}
$$

Using (5.18) for $z^{\prime}=z$ and $-z$ and (3.2), we are led to

$$
\begin{aligned}
T_{3} \leq & \tilde{C}_{\mu} L_{2}+L_{1} \int_{B_{\delta} \backslash \mathcal{C}}(\varepsilon-\delta)^{\alpha-2}|z|^{2}|\mu|(d z)+2 \alpha L_{1} \varepsilon^{\alpha-1} \int_{B \backslash B_{\delta}}|z||\mu|(d z) \\
\leq & \tilde{C}_{\mu} L_{2}+\left(1-v_{0}\right)^{\alpha-2} L_{1} \varepsilon^{\alpha-2} \int_{B_{\delta} \backslash \mathcal{C}}|z|^{2}\left|\mu_{\bar{x}}-\mu_{\bar{y}}\right|(d z) \\
& +2 \alpha L_{1} \varepsilon^{\alpha-1} \int_{B \backslash B_{\delta}}|z|\left|\mu_{\bar{x}}-\mu_{\bar{y}}\right|(d z),
\end{aligned}
$$

where $|\mu|=\mu^{+}+\mu^{-}=\left|\mu_{\bar{x}}-\mu_{\bar{y}}\right|$.

We now use (3.3) and (3.4). It is convenient to introduce

$$
\psi_{\beta}(r)= \begin{cases}r^{1-\beta} & \text { if } \beta \neq 1 \\ |\ln r| & \text { if } \beta=1\end{cases}
$$

We finally get

$$
T_{3} \leq \tilde{C}_{\mu} L_{2}+v_{0}^{\alpha-\beta} L_{1} \omega_{\mu}(\varepsilon) \varepsilon^{\alpha-\beta}+2 \alpha L_{1} \omega_{\mu}(\varepsilon) \varepsilon^{\alpha-1} \psi_{\beta}\left(v_{0} \varepsilon\right)
$$

for $v_{0}$ small enough. We use here the fact that $v_{0}^{\alpha-\beta}$ controls $\left(1-v_{0}\right)^{\alpha-2} v_{0}^{2-\beta}$ from above. 
Final estimate for $T_{n l}$. Combining [5.9, 5.15, 5.19, the final estimates are: for $\beta \neq 1$,

$$
T_{n l} \leq-L_{1} C_{5} \varepsilon^{\alpha-\beta}+o\left(\varepsilon^{\alpha-\beta}\right)+O\left(L_{2}\right),
$$

and for $\beta=1$,

$$
T_{n l} \leq-L_{1} C_{5} \varepsilon^{\alpha-1}+v_{0}^{\alpha-1} L_{1} \omega_{\mu}(\varepsilon) \varepsilon^{\alpha-1}+2 \alpha L_{1} \omega_{\mu}(\varepsilon) \varepsilon^{\alpha-1}\left|\ln \left(v_{0} \varepsilon\right)\right|+O\left(L_{2}\right) .
$$

We see that if $\omega_{\mu}(r)$ satisfies $\omega_{\mu}(r)|\ln r| \rightarrow 0$ as $r \rightarrow 0$ (which is the case when $\beta=1$ ), (5.20) still holds true in this case.

Step 4: Conclusion. For all $\alpha<1$, we deduce from 5.20 that $T_{n l} \leq 0$ if $L_{1}$ is large enough. Using inequality (5.4) together with $(\mathrm{H})$ with $L=R=O\left(L_{2}\right)$ and estimates (5.8) \& (5.20), and recalling that $\bar{\varepsilon}:=\left(L_{1} \alpha \varepsilon^{\alpha-2}\right)^{-1}$, we are thus led to

$$
0 \leq \Lambda_{1}(\bar{x}) A_{1}+\Lambda_{2}(\bar{x}) A_{2}
$$

with

$$
\begin{aligned}
& A_{1}=L_{1} \alpha \varepsilon^{\alpha-2}\left[-8 \varpi+\omega_{F}(\varepsilon)\right]+\varepsilon^{\tau}\left(\alpha L_{1} \varepsilon^{\alpha-1}\right)^{2+\tau}+\left(\alpha L_{1} \varepsilon^{\alpha-1}\right)^{2}+\tilde{\chi}\left(L_{2}\right), \\
& A_{2}=\left[-L_{1} C_{5} \varepsilon^{\alpha-\beta}+o\left(\varepsilon^{\alpha-\beta}\right)\right]+\frac{\varepsilon^{2 \theta}}{\bar{\varepsilon}}+\varepsilon^{\tau}\left(\alpha L_{1} \varepsilon^{\alpha-1}\right)^{k+\tau}+\left(\alpha L_{1} \varepsilon^{\alpha-1}\right)^{k}+\tilde{\chi}\left(L_{2}\right),
\end{aligned}
$$

where we have gathered in $\tilde{\chi}\left(L_{2}\right)$ the terms that either depend on $L_{2}$ or are bounded. We use the assumption $\Lambda_{1}+\Lambda_{2} \geq \Lambda_{0}>0$ by rewriting (5.21) as follows:

$$
0 \leq \Lambda_{0} \max \left(A_{1}, A_{2}\right) \text {. }
$$

To get the desired contradiction and to obtain the $C^{0, \alpha}$-estimate, it is enough to prove that $A_{i}<0$ for $i=1,2$ and $L_{1}$ large enough, and to control the size of such $L_{1}$.

As far as $A_{1}$ is concerned and as soon as $\alpha<1$, we can ensure that $-8 \varpi+\omega_{F}(\varepsilon) \leq$ $-4 \varpi$ if $L_{1}$ is large enough; using (5.1), it is clear that, in order to do it, the size of $L_{1}$ depends only on $\|u\|_{\infty}, \alpha$ and $d\left(x_{0}, \partial \Omega\right)$. This yields an estimate of the type

$$
A_{1} \leq L_{1} \varepsilon^{\alpha-2}\left[-4 \varpi \alpha+\alpha^{2+\tau}\left(L_{1} \varepsilon^{\alpha}\right)^{1+\tau}+\alpha^{2} L_{1} \varepsilon^{\alpha}\right]+\tilde{\chi}\left(L_{2}\right) .
$$

Now there are two ways to estimate $L_{1} \varepsilon^{\alpha}$ : either to use the first part of inequality (5.2) which yields the estimate $L_{1} \varepsilon^{\alpha} \leq 2\|u\|_{\infty}$, or to use the second part and the estimate of $L_{1} \varepsilon^{\alpha}$ through the modulus of continuity of $u$. The strategy of the proof consists in proving the result for $\alpha$ small enough by using the first estimate of $L_{1} \varepsilon^{\alpha}$ and then to use this first step (which provides a modulus of continuity of $u$ ) to prove it for all $\alpha$ by using the second estimate of $L_{1} \varepsilon^{\alpha}$.

Using $L_{1} \varepsilon^{\alpha} \leq 2\|u\|_{\infty}$ in the above inequality yields

$$
A_{1} \leq L_{1} \varepsilon^{\alpha-2}\left[-4 \varpi \alpha+\alpha^{2+\tau}\left(2\|u\|_{\infty}\right)^{1+\tau}+2 \alpha^{2}\|u\|_{\infty}\right]+\tilde{\chi}\left(L_{2}\right) .
$$

For $\alpha$ small enough (depending only on $\|u\|_{\infty}$ and $\tau$ ), the bracket is less than $-2 \varpi \alpha<0$ and, recalling 5.1, it is clear that the right-hand side is (strictly) negative if $L_{1}$ is large enough (depending on $\alpha, \varpi, \tilde{\chi}\left(L_{2}\right)$ ). Hence, we get the desired inequality: $A_{1}<0$. 
For the $A_{2}$-term, we first write

$$
\begin{aligned}
A_{2}= & -L_{1} C_{5} \varepsilon^{\alpha-\beta}+o\left(\varepsilon^{\alpha-\beta}\right)+\varepsilon^{2 \theta} L_{1} \varepsilon^{\alpha-2}+\varepsilon^{\tau}\left(\alpha L_{1} \varepsilon^{\alpha-1}\right)^{k+\tau}+\left(L_{1} \alpha \varepsilon^{\alpha-1}\right)^{k} \\
& +\tilde{\chi}\left(L_{2}\right) \\
= & L_{1} \varepsilon^{\alpha-\beta}\left[-C_{5}+o_{\varepsilon}(1)+\varepsilon^{2 \theta-2+\beta}+\alpha^{k+\tau} \varepsilon^{\beta-k}\left(L_{1} \varepsilon^{\alpha}\right)^{k+\tau-1}\right. \\
& \left.+\alpha^{k} \varepsilon^{\beta-k}\left(L_{1} \varepsilon^{\alpha}\right)^{k-1}\right]+\tilde{\chi}\left(L_{2}\right) \\
= & L_{1} \varepsilon^{\alpha-\beta}\left[-C_{5}+o_{\varepsilon}(1)+\alpha^{k+\tau} \varepsilon^{\beta-k}\left(L_{1} \varepsilon^{\alpha}\right)^{k+\tau-1}+\alpha^{k} \varepsilon^{\beta-k}\left(L_{1} \varepsilon^{\alpha}\right)^{k-1}\right]+\tilde{\chi}\left(L_{2}\right)
\end{aligned}
$$

where $C_{5}$ is given by 5.16; we also used $2 \theta+\beta-2>0$.

The key difference from $A_{1}$ is the fact that the exponents of the term $L_{1} \varepsilon^{\alpha}$ can be non-positive and we have to argue differently if it is indeed the case.

We have the following cases.

- If $\beta>1$, since $C_{5} \geq \alpha C_{5}^{\prime}$ for some constant $C_{5}^{\prime}$ independent of $\alpha$ (at least for $\alpha \leq 1 / 2$ ), then one can argue as for $A_{1}$ with $k=\beta$ since $k+\tau>1$ and $k=\beta>1$ and obtain the $C^{0, \alpha}$ regularity and estimates for $\alpha$ small enough.

- If $\beta \leq 1$, then we cannot use this argument anymore since $k$ must satisfy (at least) $k \leq 1$. In order to conclude, it is enough to ensure

$$
\varepsilon^{\beta-k}\left(L_{1} \varepsilon^{\alpha}\right)^{k+\tau-1}=o_{\varepsilon}(1) \quad \text { and } \quad \varepsilon^{\beta-k}\left(L_{1} \varepsilon^{\alpha}\right)^{k-1}=o_{\varepsilon}(1) .
$$

Writing $\varepsilon^{\beta-k}\left(L_{1} \varepsilon^{\alpha}\right)^{k-1}=L_{1}^{k-1} \varepsilon^{\beta-k+\alpha(k-1)}$, we see that this term is $o_{\varepsilon}(1)$ if $k<1$ and $\beta-k+\alpha(k-1) \geq 0$; notice that we do not know how to compare, in full generality, $L_{1}$-terms and $\varepsilon$-terms. The second condition implies that $k<\beta$. In the same way, for the other term, either $k+\tau-1 \geq 0$ and the condition $\beta>k$ is sufficient, or $k+\tau-1<0$ and we are led to $\alpha \leq(\beta-k) /(1-k-\tau)$. Gathering all this information yields the conditions

$$
1 \geq \beta>k \quad \text { and } \quad \alpha \leq \frac{\beta-k}{1-k} .
$$

At this point, putting together the information on $A_{1}$ and $A_{2}$, we have shown that $u$ is locally in $C^{0, \bar{\alpha}}$ for $\bar{\alpha}$ small enough (depending only on the data and the $L^{\infty}$-norm of $u$ ) and we have an estimate of the local $C^{0, \bar{\alpha}}$-norm of $u$. In order to conclude the proof, we need to go back to the estimate on $A_{1}$ and $A_{2}$ (in the case when $\beta>1$ ) and to estimate the terms $L_{1} \varepsilon^{\alpha}$ using (5.2) and the local $C^{0, \bar{\alpha}}$-modulus of continuity. This easily yields the full result and the proof of (i) is complete.

In order to prove the second part of the theorem, we now choose $\alpha=\beta$ and we only need to adapt the final step (Step 4) in the previous proof. We proceed as before by writing 5.21) with $A_{1}$ unchanged and, since $C_{F}=0$, with $A_{2}$ given by

$$
A_{2}=L_{1}\left[-C_{5}+o(1)\right]+L_{1} \varepsilon^{\beta-2+2 \theta}+\varepsilon^{\tau}\left(\beta L_{1} \varepsilon^{\beta-1}\right)^{k+\tau}+\tilde{\chi}\left(L_{2}\right),
$$

which we can rewrite as

$$
A_{2}=L_{1}\left[-C_{5}+o(1)\right]+L_{1} \varepsilon^{\beta-2+2 \theta}+\beta L_{1} \varepsilon^{\tau+(\beta-1)(k+\tau)}+\tilde{\chi}\left(L_{2}\right) .
$$


At this stage of the proof, $L_{2}$ is fixed and $L_{1}$ can be chosen large enough in order to control the term $\tilde{\chi}\left(L_{2}\right)$. Next we notice that $\beta-2+2 \theta>0$. In order to conclude, it is enough to have $\tau+(\beta-1)(k+\tau)>0$, i.e. $\tau>k\left(\beta^{-1}-1\right)$.

The proof of Theorem 3.1 is now complete.

\subsection{Proof of Theorem 3.2}

This proof follows the lines of the previous one, the only difference is the way of getting estimate 5.20 for the non-local term in the new framework and under the new assumptions. Let us explain this point.

In order to shed light on the fact that $j$ has to be Hölder continuous with respect to $x$, we let $\omega(r)$ denote $C_{0} r^{\tilde{\theta}}$ and we will see at the end of the present proof that $\omega$ has to be chosen as a power law. More precisely, in this case,

$$
\begin{aligned}
T_{n l}= & \int_{|z| \geq \delta^{\prime}}\left[u(\bar{x}+j(\bar{x}, z))-u(\bar{x})-(p+q) \cdot j(\bar{x}, z) \mathbf{1}_{B}(z)\right] \mu(d z) \\
& -\int_{|z| \geq \delta^{\prime}}\left[u(\bar{y}+j(\bar{y}, z))-u(\bar{y})-p \cdot j(\bar{y}, z) \mathbf{1}_{B}(z)\right] \mu(d z) .
\end{aligned}
$$

We then write $T_{n l}=T_{1}+T_{2}+T_{3}$ with

$$
\begin{aligned}
T_{1}= & \int_{|z| \geq 1}[u(\bar{x}+j(\bar{x}, z))-u(\bar{x})] \mu(d z)-\int_{|z| \geq 1}[u(\bar{y}+j(\bar{y}, z))-u(\bar{y})] \mu(d z), \\
T_{2}= & \int_{z \in \mathcal{C}}[u(\bar{x}+j(\bar{x}, z))-u(\bar{x})-(p+q) \cdot j(\bar{x}, z)] \mu(d z) \\
& -\int_{z \in \mathcal{C}}[u(\bar{y}+j(\bar{y}, z))-u(\bar{y})-p \cdot j(\bar{y}, z)] \mu(d z), \\
T_{3}= & \int_{z \in B, z \notin \mathcal{C}}[\ldots] \mu_{\bar{x}}(d z)-\int_{z \in B, z \notin \mathcal{C}}[\ldots] \mu_{\bar{y}}(d z),
\end{aligned}
$$

where $\mathcal{C}$ is defined in the following way:

$$
\begin{aligned}
\mathcal{C} & :=\left\{z:\left|j\left(\frac{\bar{x}+\bar{y}}{2}, z\right)\right| \leq \frac{\delta}{2} \text { and }\left|j\left(\frac{\bar{x}+\bar{y}}{2}, z\right) \cdot \hat{a}\right| \geq\left(1-\frac{\eta}{2}\right)\left|j\left(\frac{\bar{x}+\bar{y}}{2}, z\right)\right|\right\} \\
& =\mathcal{C}_{\delta / 2, \eta / 2}\left(\frac{\bar{x}+\bar{y}}{2}\right),
\end{aligned}
$$

where the notation $\mathcal{C}_{\delta, \eta}(x)$ is defined in the statement of Theorem 3.2 Roughly speaking, $\mathcal{C}$ is the analogue of the cone used in the proof of Theorem 3.1 where we have replaced $z$ by $j((\bar{x}+\bar{y}) / 2, z)$. Notice that, because of 3.5$), \mathcal{C} \subset B$ if $\delta$ is small enough.

We have chosen such a set $\mathcal{C}$ for the following reason: if $L_{1}$ is large enough (or equivalently $\varepsilon$ or $\delta$ is small enough), then

$$
\mathcal{C} \subset \mathcal{C}_{\delta, \eta}(\bar{x}) \cap \mathcal{C}_{\delta, \eta}(\bar{y})
$$

which means that both $j(\bar{x}, z)$ and $j(\bar{y}, z)$ are in the "good" cones. 
To check these properties, we write

$$
\begin{aligned}
|j(\bar{x}, z) \cdot \hat{a}| & \geq\left|j\left(\frac{\bar{x}+\bar{y}}{2}, z\right) \cdot \hat{a}\right|-\left|j(\bar{x}, z)-j\left(\frac{\bar{x}+\bar{y}}{2}, z\right)\right| \\
& \geq(1-\eta / 2)\left|j\left(\frac{\bar{x}+\bar{y}}{2}, z\right)\right|-|z| \omega\left(\left|\bar{x}-\frac{\bar{x}+\bar{y}}{2}\right|\right) \\
& \geq(1-\eta / 2)|j(\bar{x}, z)|-(2-\eta / 2)|z| \omega(\varepsilon / 2) \\
& \geq\left(1-\eta / 2-(2-\eta) C_{0}^{-1} \omega(\varepsilon / 2)\right)|j(\bar{x}, z)| \geq(1-\eta)|j(\bar{x}, z)|
\end{aligned}
$$

and

$$
|j(\bar{x}, z)| \leq\left|j\left(\frac{\bar{x}+\bar{y}}{2}, z\right)\right|+\omega(\varepsilon / 2)|z| \leq \frac{\delta}{2}+\frac{\delta}{2 c_{0}} \omega(\varepsilon / 2) \leq \delta
$$

for $L_{1}$ such that

$$
\omega(\varepsilon / 2) \leq \min \left(\frac{\eta c_{0}}{4-\eta}, c_{0}\right)=\min \left(C_{0}, c_{0}\right)=c_{0}
$$

for $\eta<1$.

Estimate of $T_{1}$. We remark that, thanks to the properties of $j$ and $\mu$, 5.9) still holds true.

Estimate of $T_{2}$. One can check that (5.14) and (5.15) still hold true. Indeed, we use (3.6) (3.7) in order to get (3.2). More precisely, using (3.6) - 3.7) and recalling the computation we made in Subsection 4.3 , we obtain

$$
\forall \hat{a}, \quad \int_{\mathcal{C}}|z|^{2} \mu(d z) \geq C_{\mu} \delta^{2-\beta}
$$

where $C_{\mu}=C\left(c_{0}, C_{0}, C_{\mu}^{-}, \eta, d, \beta\right)$. Notice that 5.12 is slightly modified and so is $C_{4}$ (and consequently $C_{5}$ ).

Estimate of $T_{3}$. In the case of Lévy-Itô operators, we estimate $T_{3}$ as follows. By using 5.11) with $d=j(\bar{x}, z)$ and $d^{\prime}=j(\bar{y}, z)$, we get

$$
\begin{aligned}
T_{3}=\int_{z \in B, z \notin \mathcal{C}}[u(\bar{x}+ & j(\bar{x}, z))-u(\bar{x})-(p+q) \cdot j(\bar{x}, z) \\
& -u(\bar{y}+j(\bar{y}, z))+u(\bar{y})+p \cdot j(\bar{y}, z)] \mu(d z) \leq T_{3}^{1}+T_{3}^{2}
\end{aligned}
$$

with

$$
\begin{aligned}
T_{3}^{1} & =\int_{z \in B, z \notin \mathcal{C}}[\Gamma(\bar{x}+j(\bar{x}, z))-\Gamma(\bar{x})-q \cdot j(\bar{x}, z)] \mu(d z), \\
T_{3}^{2} & =\int_{z \in B, z \notin \mathcal{C}}[\phi(a+\Delta(z))-\phi(a)-p \cdot \Delta(z)] \mu(d z),
\end{aligned}
$$

where $\Delta(z)=j(\bar{x}, z)-j(\bar{y}, z)$. Let us first estimate $T_{3}^{1}$ as follows:

$$
T_{3}^{1} \leq \frac{1}{2} \int_{B} \sup _{t \in(0,1)}\left[D^{2} \Gamma(\bar{x}+t j(\bar{x}, z)) j(\bar{x}, z) \cdot j(\bar{x}, z)\right] \mu(d z)
$$


and we deduce that

$$
T_{3}^{1} \leq C_{2} L_{2}
$$

where

$$
C_{2}=C_{0}^{2} \int_{|z| \leq 1}|z|^{2} \mu(d z) .
$$

We now turn to $T_{3}^{2}$ and we write $T_{3}^{2}=T_{3}^{2,1}+T_{3}^{2,2}$ with

$$
\begin{aligned}
T_{3}^{2,1} & =\int_{z \in B, z \notin \mathcal{C},|\Delta(z)| \geq \delta}[\phi(a+\Delta(z))-\phi(a)-D \phi(a) \cdot \Delta(z)] \mu(d z), \\
T_{3}^{2,2} & =\int_{z \in B, z \notin \mathcal{C},|\Delta(z)| \leq \delta}[\phi(a+\Delta(z))-\phi(a)-D \phi(a) \cdot \Delta(z)] \mu(d z) .
\end{aligned}
$$

We can now estimate $T_{3}^{2,1}$. In order to clarify computations, we write $\omega$ for $\omega(\varepsilon)$ in the following.

We use 5.18; ; remarking that $\delta \leq|\Delta(z)| \leq \omega|z|$, we deduce

$$
\begin{aligned}
T_{3}^{2,1} & \leq \int_{\delta \omega^{-1} \leq|z| \leq 1} 2 \alpha L_{1} \varepsilon^{\alpha-1}|\Delta(z)| \mu(d z) \leq \int_{\delta \omega^{-1} \leq|z| \leq 1} 2 \alpha L_{1} \varepsilon^{\alpha-1} \omega|z| \mu(d z) \\
& \leq 2 \alpha L_{1} \varepsilon^{\alpha-1} \omega \psi_{\beta}\left(\delta \omega^{-1}\right)
\end{aligned}
$$

where, by (3.7),

$$
\psi_{\beta}(r)=\int_{r \leq|z| \leq 1}|z| \mu(d z) \leq \begin{cases}\tilde{C}_{\mu} r^{1-\beta} & \text { if } \beta \neq 1 \\ \tilde{C}_{\mu}|\ln r| & \text { if } \beta=1 .\end{cases}
$$

In order to estimate $T_{3}^{2,2}$, we use a Taylor expansion together with 5.7 and the fact that $|a+t \Delta(z)| \geq \varepsilon-\delta>0$. It follows that

$$
\begin{aligned}
T_{3}^{2,2} & \leq \frac{L_{1} \alpha}{2} \int_{|z| \leq 1} \sup _{t \in(0,1)}|a+t \Delta(z)|^{\alpha-2}|\Delta(z)|^{2} \mu(d z) \leq \frac{L_{1}}{2} \int_{B}|z|^{2} \mu(d z)(\varepsilon-\delta)^{\alpha-2} \omega^{2} \\
& \leq \frac{L_{1}}{2} \omega^{2} \varepsilon^{\alpha-2}\left(1-v_{0}\right)^{\alpha-2} \int_{B}|z|^{2} \mu(d z) .
\end{aligned}
$$

Gathering the estimates on $T_{3}^{2,1}$ and $T_{3}^{2,2}$, we obtain

$$
T_{3}^{2} \leq 2 \alpha L_{1} \varepsilon^{\alpha-1} \omega \psi_{\beta}\left(\delta \omega^{-1}\right)+L_{1} C_{3} \omega^{2} \varepsilon^{\alpha-2}
$$

with

$$
C_{3}=\frac{1}{2} \int_{B}|z|^{2} \mu(d z)\left(1-v_{0}\right)^{\alpha-2}
$$

Final estimate of $T_{n l}$. Gathering (5.9), 5.15), 5.24) and (5.27), we finally obtain

$$
T_{n l} \leq 2 \alpha L_{1} \omega \psi_{\beta}\left(v_{0} \omega^{-1} \varepsilon\right) \varepsilon^{\alpha-1}+L_{1} C_{3} \omega^{2} \varepsilon^{\alpha-2}-L_{1} C_{5} \varepsilon^{\alpha-\beta}+O\left(L_{2}\right) .
$$


Looking at the $\omega^{2} \varepsilon^{\alpha-2}$-term, we see that we need to assume that $j$ is Hölder continuous with respect to $x$ and we thus replace $\omega(\varepsilon)$ with $C \varepsilon^{\tilde{\theta}}$. Denoting $C_{3}^{\prime}=C C_{3}$, we get

$$
T_{n l} \leq 2 \alpha L_{1} \varepsilon^{\alpha+\tilde{\theta}-1} \psi_{\beta}\left(v_{0} \varepsilon^{1-\tilde{\theta}}\right)+L_{1} C_{3}^{\prime} \varepsilon^{\alpha-2+2 \tilde{\theta}}-L_{1} C_{5} \varepsilon^{\alpha-\beta}+O\left(L_{2}\right) .
$$

We claim that as in the case of Theorem 3.1. 5.20) holds true under the assumptions of Theorem 3.2. To see this, we write

$$
\begin{aligned}
2 \alpha L_{1} \varepsilon^{\alpha+\tilde{\theta}-1} \psi_{\beta}\left(v_{0} \varepsilon^{1-\tilde{\theta}}\right)+L_{1} \varepsilon^{\alpha-2+2 \tilde{\theta}} & =L_{1} \varepsilon^{\alpha-\beta}\left[\varepsilon^{\beta+\tilde{\theta}-1} \psi_{\beta}\left(\nu_{0} \varepsilon^{1-\tilde{\theta}}\right)+C_{3}^{\prime} \varepsilon^{\beta-2+2 \tilde{\theta}}\right] \\
& =L_{1} \varepsilon^{\alpha-\beta}\left[\varepsilon^{\beta+\tilde{\theta}-1} \psi_{\beta}\left(\nu_{0} \varepsilon^{1-\tilde{\theta}}\right)+o_{\varepsilon}(1)\right]
\end{aligned}
$$

since $\tilde{\theta}>\frac{1}{2}(2-\beta)$ and $\alpha<\beta$. We next distinguish two cases.

- If $\beta \neq 1$, then by (5.26), we get

$$
\varepsilon^{\beta+\tilde{\theta}-1} \psi_{1}\left(\nu_{0} \varepsilon^{1-\tilde{\theta}}\right)=O\left(\varepsilon^{\tilde{\theta} \beta}\right)
$$

and we conclude in this case.

- If $\beta=1$,

$$
\varepsilon^{\beta+\tilde{\theta}-1} \psi_{1}\left(\nu_{0} \varepsilon^{1-\tilde{\theta}}\right)=O\left(\varepsilon^{\tilde{\theta}}\left|\ln \left(\varepsilon^{1-\tilde{\theta}}\right)\right|\right)=o_{\varepsilon}(1)
$$

and we can conclude in this case too.

The proof of Theorem 3.2 is now complete.

\section{Appendix}

In this Appendix we provide the explicit computation of $Z^{\bar{\varepsilon} / 2}$ used in the proof of Theorem 3.1 .

We have $Z=(1 / \bar{\varepsilon})(I-(2-\alpha) \hat{a} \otimes \hat{a})$ and

$$
Z^{\bar{\varepsilon} / 2} q \cdot q=\sup _{p \in \mathbb{R}^{N}}\left\{Z p \cdot p-\frac{2}{\bar{\varepsilon}}|p-q|^{2}\right\}=\frac{2}{\bar{\varepsilon}} \sup _{p \in \mathbb{R}^{N}}\left\{\frac{\bar{\varepsilon}}{2} Z p \cdot p-|p-q|^{2}\right\} .
$$

One checks easily that the (last) sup is achieved at a point $p$ such that $\bar{\varepsilon} Z p=2(p-q)$, i.e.

$$
p-(2-\alpha)(\hat{a} \cdot p) \hat{a}=2(p-q) .
$$

Taking a scalar product with $\hat{a}$, we deduce

$$
\hat{a} \cdot p=\frac{2}{3-\alpha} \hat{a} \cdot q
$$

and inserting in the previous equality yields

$$
p-q=q-\frac{2(2-\alpha)}{3-\alpha}(\hat{a} \cdot q) \hat{a} .
$$


Going back to the value of the supremum and using (5.30), we have

$$
Z^{\bar{\varepsilon} / 2} q \cdot q=\frac{2}{\bar{\varepsilon}}\left\{(p-q) \cdot p-|p-q|^{2}\right\}=\frac{2}{\bar{\varepsilon}}(p-q) \cdot q,
$$

and now using 5.31, we finally obtain

$$
Z^{\bar{\varepsilon} / 2} q \cdot q=\frac{2}{\bar{\varepsilon}}\left\{|q|^{2}-\frac{2(2-\alpha)}{3-\alpha}(\hat{a} \cdot q)^{2}\right\}
$$

Therefore $1+\varpi=2(2-\alpha) /(3-\alpha)$ and $\varpi=(1-\alpha) /(3-\alpha)$.

Acknowledgments. The authors want to thank the referees for their very careful reading of the article and for valuable suggestions which led to a real improvement of the presentation of our results. They are particularly grateful to one of the referees who provided a technical idea which allowed them to slightly weaken the assumptions of their results.

\section{References}

[1] Barles, G., Interior gradient bounds for the mean curvature equation by viscosity solutions methods. Differential Integral Equations 4, 263-275 (1991) Zbl $0735.35020 \mid$ MR 1081183

[2] Barles, G., Chasseigne, E., Imbert, C.: Dirichlet boundary conditions for second order elliptic non-linear integro-differential equations. Indiana Univ. Math. J. 57, 213-246 (2007) Zbl 1139.47057

[3] Barles, G., Da Lio, F.: Local $C^{0, \alpha}$ estimates for viscosity solutions of Neumann-type boundary value problems. J. Differential Equations 225, 202-241 (2006) Zbl 1147.35312 MR 2228697

[4] Barles, G., Imbert, C.: Second-order elliptic integro-differential equations: Viscosity solutions' theory revisited. Ann. Inst. H. Poincaré Anal. Non Linéaire 25, 567-585 (2008) Zbl 1155.45004 MR 2422079

[5] Barles, G., Souganidis, P. E.: Space-time periodic solutions and long-time behavior of solutions to quasi-linear parabolic equations. SIAM J. Math. Anal. 32, 1311-1323 (2001) Zbl 0986.35047 MR 1856250

[6] Bass, R. F., Levin, D. A.: Harnack inequalities for jump processes. Potential Anal. 17, 375388 (2002) Zbl 0997.60089 MR 1918242

[7] Caffarelli, L. A., Cabré, X.: Fully Nonlinear Elliptic Equations. Amer. Math. Soc. Colloq. Publ. 43, Amer. Math. Soc., Providence, RI (1995) Zbl 0834.35002 MR 1351007

[8] Caffarelli, L. A., Silvestre, L.: Regularity theory for fully nonlinear integro-differential equations. Comm. Pure Appl. Math. 62, 597-638 (2009) Zbl 1170.45006 MR 2494809

[9] Carr, P., Gema, H., Madan, D. B., Yor, M.: Stochastic volatility for Lévy processes. Math. Finance 13, 345-382 (2003) Zbl 1092.91022 MR 1995283

[10] Chen, Y. Z.: $C^{1, \alpha}$ regularity of viscosity solutions of fully non-linear elliptic PDE under natural structure conditions. J. Partial Differential Equations 6, 193-216 (1993) Zbl 0781.35011 MR 1234572

[11] Crandall, M. G., Ishii, H.: The maximum principle for semicontinuous functions. Differential Integral Equations 3, 1001-1014 (1990) Zbl $0723.35015 \mid$ MR 1073054

[12] Ishii, H.: On uniqueness and existence of viscosity solutions of fully nonlinear second-order elliptic PDEs. Comm. Pure Appl. Math. 42, 15-45 (1989) Zbl 0645.35025 |MR 0973743 
[13] Ishii, H., Lions, P. L.: Viscosity solutions of fully non-linear second-order elliptic partial differential equations. J. Differential Equations 83, 26-78 (1990) Zbl 0708.35031 MR 1031377

[14] Jakobsen, E. R., Karlsen, K. H.: Continuous dependence estimates for viscosity solutions of integro-PDEs. J. Differential Equations 212, 278-318 (2005) Zbl 1082.45008 MR 2129093

[15] Silvestre, L.: Hölder estimates for solutions of integro-differential equations like the fractional Laplace. Indiana Univ. Math. J. 55, 1155-1174 (2006) Zbl 1101.45004 MR 2244602

[16] Trudinger, N.: Comparison principles and pointwise estimates for viscosity solutions of non-linear elliptic equations. Rev. Mat. Iberoamer. 4, 453-468 (1988) Zbl 0695.35007 MR 1048584

[17] Trudinger, N.: On regularity and existence of viscosity solutions of non-linear second order, elliptic equations. In: Partial Differential Equations and the Calculus of Variations, Vol. II, Progr. Nonlinear Differential Equations Appl. 2, Birkhäuser Boston, Boston, MA, 939-957 (1989) Zbl 0698.35056 MR 1034037 